RESEARCH ARTICLE

\title{
High-resolution X-ray flash radiography of Ti characteristic lines with multilayer Kirkpatrick-Baez microscope at the Shenguang-II Update laser facility
}

\author{
Shengzhen $\mathrm{Yi}^{1,2}$, Feng Zhang ${ }^{3}$, Qiushi Huang ${ }^{1,2}$, Lai Wei ${ }^{3}$, Yuqiu Gu ${ }^{3}$, and Zhanshan Wang ${ }^{1,2}$ \\ ${ }^{1}$ MOE Key Laboratory of Advanced Micro-Structured Materials, Tongji University, Shanghai 200092, China \\ ${ }^{2}$ School of Physics Science and Engineering, Tongji University, Shanghai 200092, China \\ ${ }^{3}$ Research Center of Laser Fusion, China Academy of Engineering Physics, Mianyang 621900, China \\ (Received 8 March 2021; revised 29 April 2021; accepted 17 May 2021)
}

\begin{abstract}
High-resolution X-ray flash radiography of Ti characteristic lines with a multilayer Kirkpatrick-Baez microscope was developed on the Shenguang-II (SG-II) Update laser facility. The microscope uses an optimized multilayer design of $\mathrm{Co} / \mathrm{C}$ and W/C stacks to obtain a high reflection efficiency of the Ti characteristic lines while meeting the precise alignment requirement at the $\mathrm{Cu} \mathrm{K} \alpha$ line. The alignment method based on dual simulated balls was proposed herein, which simultaneously realizes an accurate indication of the center field of view and the backlighter position. The optical design, multilayer coatings, and alignment method of the microscope and the experimental result of Ti flash radiography of the Au-coned $\mathrm{CH}$ shell target on the SG-II Update are described.
\end{abstract}

Keywords: flash radiography; Kirkpatrick-Baez microscope; plasma diagnostics; X-ray imaging; X-ray multilayer

\section{Introduction}

$\mathrm{X}$-ray self-emission or backlight imaging is a basic diagnostic method for characterizing the plasma state and its evolutionary behavior in the fields of high-energy-density physics (HEDP), inertial confinement fusion and laboratory astrophysics $^{[1,2]}$. The development of a high-spatial resolution $\mathrm{K} \alpha$ flash radiography method is of great scientific significance to the diagnostics of the spatial distribution of small-sized dense plasma, such as hot-spot self-emission and plasma density ${ }^{[3]}$.

Unlike long-pulse laser drives of the order of nanoseconds, short-pulse imaging diagnostics generally uses an X-ray optical system in a single-channel mode. The temporal resolution is achieved by changing the timing of the short-pulse laser. Point projection radiography and spherical curved crystal are the main diagnostic techniques for $\mathrm{K} \alpha$ flash photography. The former utilizes a micro-focus $\mathrm{K} \alpha$ backlighter generated by the interaction of a short pulse with a wire or flag target

Correspondence to: Z. Wang, MOE Key Laboratory of Advanced Micro-Structured Materials, Tongji University, Shanghai 200092, China. Email: wangzs@tongji.edu.cn as a probe to project the sample on the detector ${ }^{[4]}$. The illumination field of view is determined by the detector size, and the spatial resolution depends on the micro-focus size. To obtain a higher spatial resolution, the wire or flag target material with $10 \mu \mathrm{m}$ wire diameter is generally selected, and the spatial resolution of 10-20 $\mu \mathrm{m}$ can be achieved. Morace et al ${ }^{[5]}$ used a $1 \mathrm{ps}$ laser focused on a $10-\mu \mathrm{m}$-diameter copper wire for $\mathrm{K} \alpha$ flash photography in the LULI2000 facility, and the spatial resolution was approximately $11 \mu \mathrm{m}$. The spherical curved crystal based on the Rowland circle structure can realize monochromatic X-ray imaging in the millimeter-level field of view, with the spatial resolution of 5-15 $\mu \mathrm{m}$. The $\mathrm{K} \alpha$ characteristic lines of $\mathrm{Ti}, \mathrm{Cu}$, and $\mathrm{Zr}$ are commonly used in current HEDP-related experiments. Sawada et al. ${ }^{[6]}$ used the LFEX laser to generate the Ti $\mathrm{K} \alpha$ line $(\sim 4.5 \mathrm{keV})$ and used an $\alpha$-quartz (2023) spherical crystal to probe the implosion compression process of the $\mathrm{CD}$ cone-shell target. The spatial resolution of the image was $12.5 \pm 2.5 \mu \mathrm{m}$. Stoceckl et al..$^{[7]}$ developed an $\alpha$ quartz (2131) spherical crystal with a spatial resolution of approximately $10 \mu \mathrm{m}$ for the OMEGA EP facility. With the help of this set of curved crystals, Jarrott et al.$^{[8]}$ performed 
$\mathrm{Cu} \mathrm{K} \alpha$ self-emission imaging and successfully measured the energy transport process of hot electrons in a highdensity fast-ignition target. Theobald et al ${ }^{[9]}$ performed flash radiography with a $\mathrm{Cu} \mathrm{K} \alpha$ backlighter on the cone-shell target and studied the changes in the areal density during the implosion compression process. In general, neither point projection nor spherical curved crystals can achieve a spatial resolution better than $5 \mu \mathrm{m}$, making it difficult to meet physical experiments with finer resolution requirements.

The Kirkpatrick-Baez (KB) microscope uses two perpendicular grazing incidence mirrors to focus $\mathrm{X}$-rays with a spatial resolution of 3-5 $\mu \mathrm{m}$ in the field of view of several hundred micrometers, which provides a promising means for capturing more detailed spatial distributions of smallsized plasma ${ }^{[10]}$. A multi-channel KB microscope for longpulse X-ray imaging is currently being developed. Pickworth et al. ${ }^{[11]}$ are developing a four-channel $\mathrm{KB}$ microscope for $\mathrm{Ge} \mathrm{He}-\alpha$ line $(10.2 \mathrm{keV})$ imaging on NIF with a spatial resolution of $4 \mu \mathrm{m}$. Marshall et al. ${ }^{[12]}$ and $\mathrm{Yi}$ et al.$^{[13]}$ independently developed 16-channel $\mathrm{KB}$ microscopes with two different optical structures and achieved good diagnostic results.

At present, the diagnostic applications of KB microscope in HEDP experiments are focused on high-resolution $\mathrm{K} \alpha$ self-emission imaging. Meanwhile, a certain spectral resolution can be achieved by coating a multilayer film structure on the reflective surface. For example, Theobald et al. ${ }^{[14]}$ used a multilayer $\mathrm{KB}$ microscope to image the $\mathrm{K} \alpha$ emission lines of the $\mathrm{Cu}$-doped $\mathrm{CD}$ spherical shell target. The optimal spatial resolution of the image reached $3 \mu \mathrm{m}$. One of the challenges in utilizing $\mathrm{KB}$ microscope on $\mathrm{X}$-ray flash radiography is how to ensure that the multilayers respond to emission lines of the backlighter target with high efficiency. In addition, how to make the small-focus backlighter effectively illuminate the field of view to be diagnosed is also one of the key problems to be solved in equipment development.
This study, aiming at the problems described previously, developed a multilayer $\mathrm{KB}$ microscope for high-resolution Ti flash radiography and self-emission imaging at the Shenguang-II (SG-II) Update laser facility. The following sections describe the details on optical design, assembly, and alignment method of the microscope. The experimental results in the laboratory and laser facility are also presented.

\section{Optical design}

The KB microscope consists of two perpendicular concave spherical mirrors (M1 and M2) in tandem (Figure 1). The imaging equation of each mirror in the meridian plane is given by

$$
\frac{1}{u}+\frac{1}{v}=\frac{1}{f}=\frac{2}{R \cdot \sin \theta}
$$

where $u$ is the object distance from the target to the mirror center, $v$ is the image distance from the mirror center to the image plane, $f$ is the focus distance, $R$ is the mirror radius of curvature, and $\theta$ is the grazing incidence angle. The magnification $M$ was approximately $10 \times$ considering the spatial resolution of the image detector. The instrument will be used for X-ray imaging of the $\mathrm{Ti}$ characteristic lines; thus, the grazing incidence angle $\theta$ was selected at approximately $1^{\circ}$ to ensure the imaging quality and the multilayer reflectivity.

The mirror length $d$ was $10 \mathrm{~mm}$, and the corresponding spatial resolution obtained by the Zemax simulation nearlinearly decreased from $2 \mu \mathrm{m}$ at the central field to near $7 \mu \mathrm{m}$ within the object field of $300 \mu \mathrm{m} \times 300 \mu \mathrm{m}$ in length and width. When choosing a shorter mirror length, a higher spatial resolution can be obtained, but the image intensity will be reduced. The manufacture error of the curvature radius $(\Delta R)$ leads to deviations of the best object field of view.

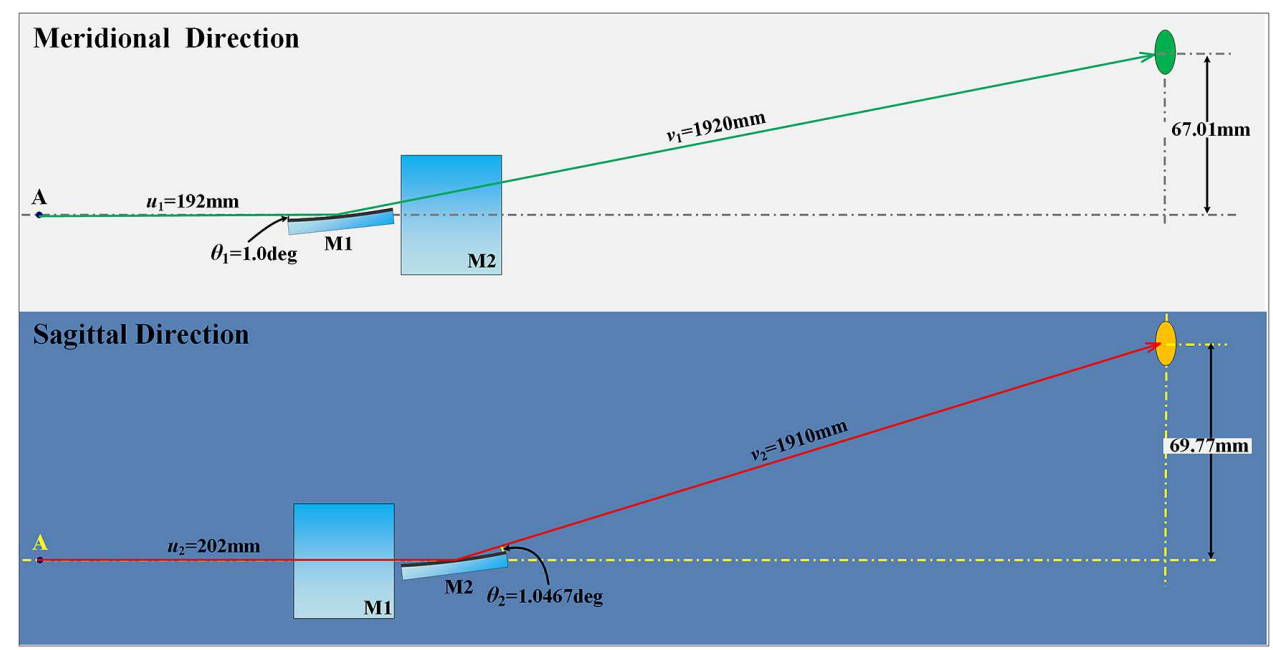

Figure 1. Schematic of the multilayer KB microscope for high-resolution Ti flash radiography. 
Table 1. Optical parameters of the multilayer KB microscope.

\begin{tabular}{lcccccc}
\hline Direction & $R(\mathrm{~m})$ & $d(\mathrm{~mm})$ & $\theta$ & $u(\mathrm{~mm})$ & $v(\mathrm{~mm})$ & $M$ \\
\hline Meridional & 19.5 & 10 & $1.000^{\circ}$ & 187.2 & 1872 & 10 \\
Sagittal & & & $1.046^{\circ}$ & 197.2 & 1862 & 9.44 \\
\hline
\end{tabular}

Although It can be compensated for by changing the actual grazing angle, the reflectivity may be seriously reduced owing to the narrow angular bandwidth of the multilayer. Thus, we optimized the following three aspects: first, the concave mirrors were accurately measured by the optical profiler (Bruker ContourGT-X3) to have a mean radius of curvature of $19.5 \mathrm{~m}$ with a root-mean-square (RMS) variation of $0.3 \mathrm{~m}$, which corresponds to the grazing angle change of $\sim 0.015^{\circ}$; second, the deviation of the grazing incidence angle at different mirror positions is within $\pm 0.025^{\circ}$ from the center value of $1.000^{\circ}$ or $1.046^{\circ}$, and therefore the multilayer bandwidth has also been properly designed to near $\pm 0.04^{\circ}$ to satisfy above grazing angle changes, as detailed in Section 3; third, according to the different grazing angles in meridian and sagittal directions, two sets of multilayer mirrors were designed and fabricated respectively. Table 1 presents the final optical parameters of the instrument.

\section{Multilayer design and fabrication}

The KB microscopes can be operated at different energy bands by coating the metal single layer or X-ray multilayer on the reflected surfaces. The metal single layers (e.g., Ir or $\mathrm{Pt}$ ) based on the total external reflection are good elements to use in the soft X-ray region. They can also respond to harder X-rays, but only at grazing angles smaller than the critical angle of the total external reflection. At this time, the lateral aberration expressed by $\delta=3 d^{2} / 8 R$ $+d q / R \sin \theta$ (where $q$ is the object field) becomes larger due to the smaller grazing angle, which reduces the spatial resolution of the $\mathrm{KB}$ microscope ${ }^{[15]}$. Compared with a metal single layer, the multilayer coatings consisting of alternating bilayers of high- $Z$ and low- $Z$ materials are a better choice to obtain a high throughput at $\mathrm{Ti}$ characteristic lines. It can be understood by the Bragg diffraction formula that $2 D \sin \theta$ $=m \lambda$, where $D$ is the periodic thickness of the multilayer, $\theta$ is the grazing angle, $m$ is the order of $\mathrm{X}$-ray diffraction; and $\lambda$ is the $\mathrm{X}$-ray wavelength. In this paper, it includes two types of multilayer stacks (i.e., $\mathrm{Co} / \mathrm{C}$ and W/C) with different parameters from the surface to the substrate. The $\mathrm{Co} / \mathrm{C}$ and $\mathrm{W} / \mathrm{C}$ are two good material pairs to obtain high reflectivity near the energy of $4.5-4.75 \mathrm{keV}$ and $8 \mathrm{keV}$ respectively because of the difference of optical constants. Among them, the $\mathrm{Co} / \mathrm{C}$ stacks were designed to have a larger spectral bandwidth by adjusting the thickness ratio of high$Z$ and low- $Z$ materials due to the existence of Ti He-like lines $(\sim 4.75 \mathrm{keV})$ and $\mathrm{K} \alpha$ lines $(\sim 4.5 \mathrm{keV})$ in the spectrum. Although the peak reflectivity was slightly reduced, the spectral bandwidth was effectively improved to cover the above two characteristic lines and obtain highest possible image intensity within a certain object field. The $\mathrm{Co} / \mathrm{C}$ stacks working at the $\mathrm{Ti}$ characteristic lines have seven bilayer numbers, whose thickness is 9.15 and $8.70 \mathrm{~nm}$ for the grazing angles of $1.000^{\circ}$ and $1.046^{\circ}$, respectively. The W/C stacks working at the $\mathrm{Cu} \mathrm{K} \alpha(\sim 8.05 \mathrm{keV})$ characteristic line will be used for the alignment and assembly of the microscope in air by a common copper tube with 30 bilayer numbers, whose thickness is 4.77 and $4.56 \mathrm{~nm}$ for the grazing angles of $1.000^{\circ}$ and $1.046^{\circ}$, respectively. In physics experiments, the Ti characteristic lines are directly reflected by the top $\mathrm{Co} / \mathrm{C}$ bilayers; hence, the bottom W/C bilayers will not reduce the X-ray throughput. The spatial resolution is only slightly different at the $\mathrm{Ti}$ characteristic lines when compared with that at $8.05 \mathrm{keV}$ owing to the $\mathrm{X}$-ray diffraction. Furthermore, under the assumption that the self-emission X-ray spectrum is of an exponential form, the influence of $8.05 \mathrm{keV} \mathrm{X}$-rays on the lower Ti characteristic lines can be negligible. All multilayers were deposited onto ultra-polished silica substrates with a DC magnetron sputtering system. The micro-roughness of the silica substrates measured by an optical profiler (Bruker Inc., CoutourGT-X3) was approximately $0.3 \mathrm{~nm}$. The control accuracy of the multilayer thicknesses with the deposition system was approximately $0.1 \mathrm{~nm}$. Figure 2 depicts the reflectivity measurements by an X-ray diffractometer (Bede $\mathrm{D}^{1}$ system) at $8.05 \mathrm{keV}$.

The spectral response of the multilayer can also be obtained by fitting the measured reflectivity curves (Figure 3). The reflectivities of both multilayers were more than $60 \%$ at 4.75 and $4.5 \mathrm{keV}$.

\section{Microscope alignment method}

The spatial resolution of the KB microscope decreased with the deviation from the central field of view because of a serious off-axis aberration. Therefore, the KB microscope must be accurately aimed to the central field of view to obtain a spatial resolution better than $5 \mu \mathrm{m}$. Meanwhile, for flash radiography, the backlighter position must be accurately adjusted to the incident optical axis of the KB microscope to ensure that the micro-focus source of less than $100 \mu \mathrm{m}$ covers the diagnostic area of interest.

We developed an accurate indication method based on dual simulated balls coupling with linear guides for the KB microscope alignment (Figure 4). First, the central field of view of the $\mathrm{KB}$ microscope (with best resolution) was found by the imaging experiment of a metal grid using a copper X-ray tube $(8.05 \mathrm{keV}, \mathrm{Cu} \mathrm{K} \alpha$ line). Figure 5(a) depicts the image of a 600 -mesh Au grid $(43 \mu \mathrm{m}$ period with 6$7 \mu \mathrm{m}$ linewidth measured by scanning electron microscopy) recorded by a phosphor X-ray CCD (Photonic Science: VHR-11M) with $4096 \times 3060$ pixels and $9.0 \times 9.0 \mu \mathrm{m}$ pixel size. The phosphor material is Gadox:Tb with the thickness 

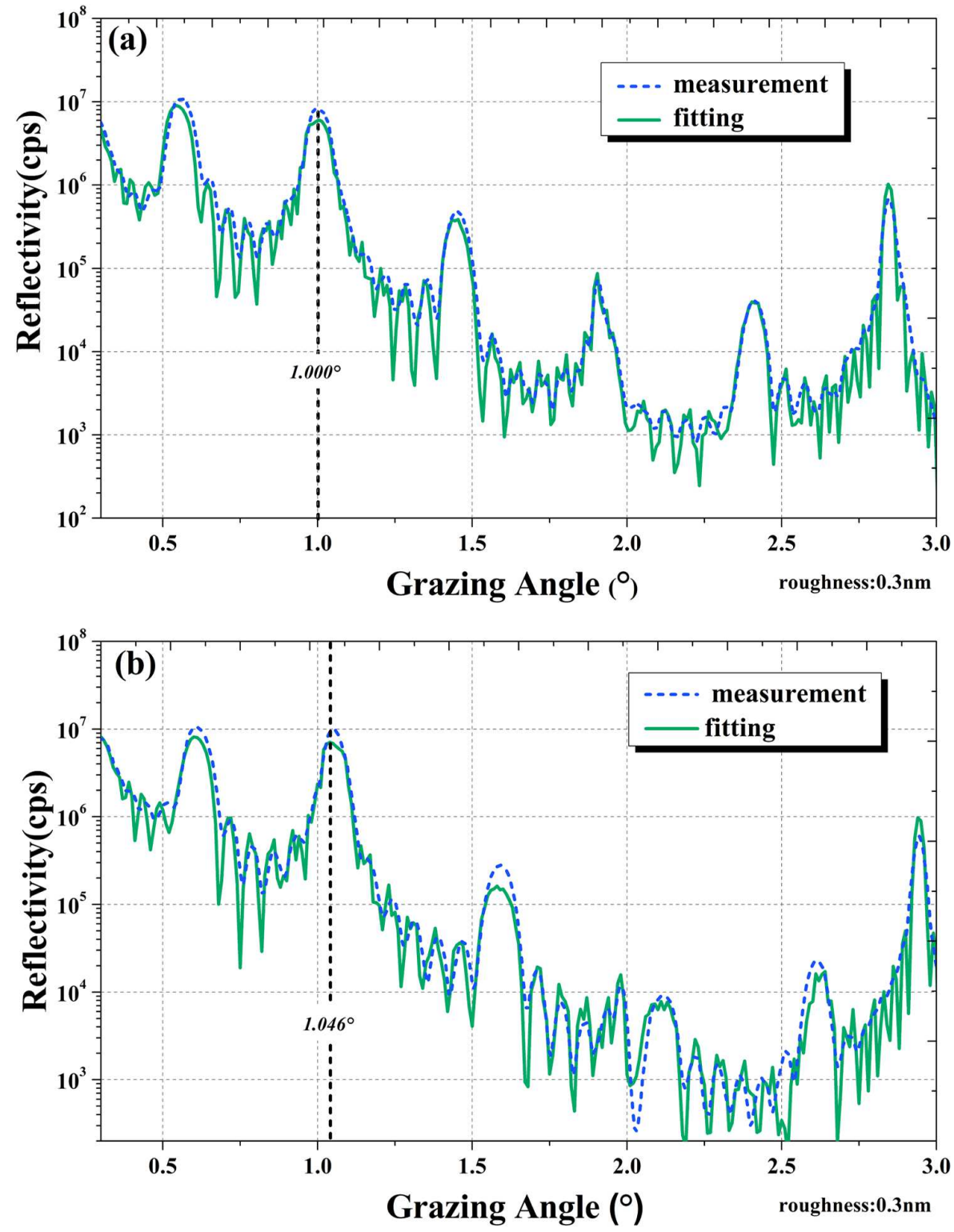

Figure 2. Reflectivity measurement results of the KB multilayer by the X-ray diffractometer for grazing angles of (a) $1.000^{\circ}$ and (b) $1.046^{\circ}$.

of $20 \mu \mathrm{m}$ and the spatial resolution of $30 \mathrm{lp} / \mathrm{mm}$. The total quantum gain at $\mathrm{Cu} \mathrm{K} \alpha$ line is 3.5 electrons per incident $\mathrm{X}$ ray photon. The exposure time was $10 \mathrm{~min}$ with the circuit gain of 50 to get enough image brightness. The hole with approximately $150 \mu \mathrm{m}$ diameter in the grid was used as a reference for the best resolution. The spatial resolution defined as the distance between $10 \%$ and $90 \%$ intensity appears in Figure 5(b) by three measurements along the horizonal dotted line in Figure 5(a), which was approximately $3.0 \mu \mathrm{m}$ in the hole center and better than $5 \mu \mathrm{m}$ in approximately $200 \mu \mathrm{m}$ field of view. The solid line and dash-dotted line represent simulation results of spatial resolution with and without the additional errors, respectively. The additional errors, which are mainly the pixel size of the X-ray CCD camera, the figure error, and the mirror roughness, reduce the actual resolution, especially for the central object field. We estimate that the reference hole is deviated by less than $20 \mu \mathrm{m}$ from the central object field, which is acceptable in terms of spatial resolution. The actual magnifications in the meridional and sagittal directions were approximately $10.15 \times$ and $9.57 \times$, respectively. Then the position of reference hole was replaced by the first simulated ball with a diameter of approximately $500 \mu \mathrm{m}$ under the monitoring of two orthogonal visible light CCD cameras. The incident optical axis of the $\mathrm{KB}$ microscope is defined with the first simulated ball and the mirror center of the $\mathrm{KB}$ microscope as two points. Finally, the second simulated ball with a diameter of approximately $300 \mu \mathrm{m}$ was placed on the incident optical axis to indicate the micro-focus source position. The distance between the two simulated balls was consistent with the 

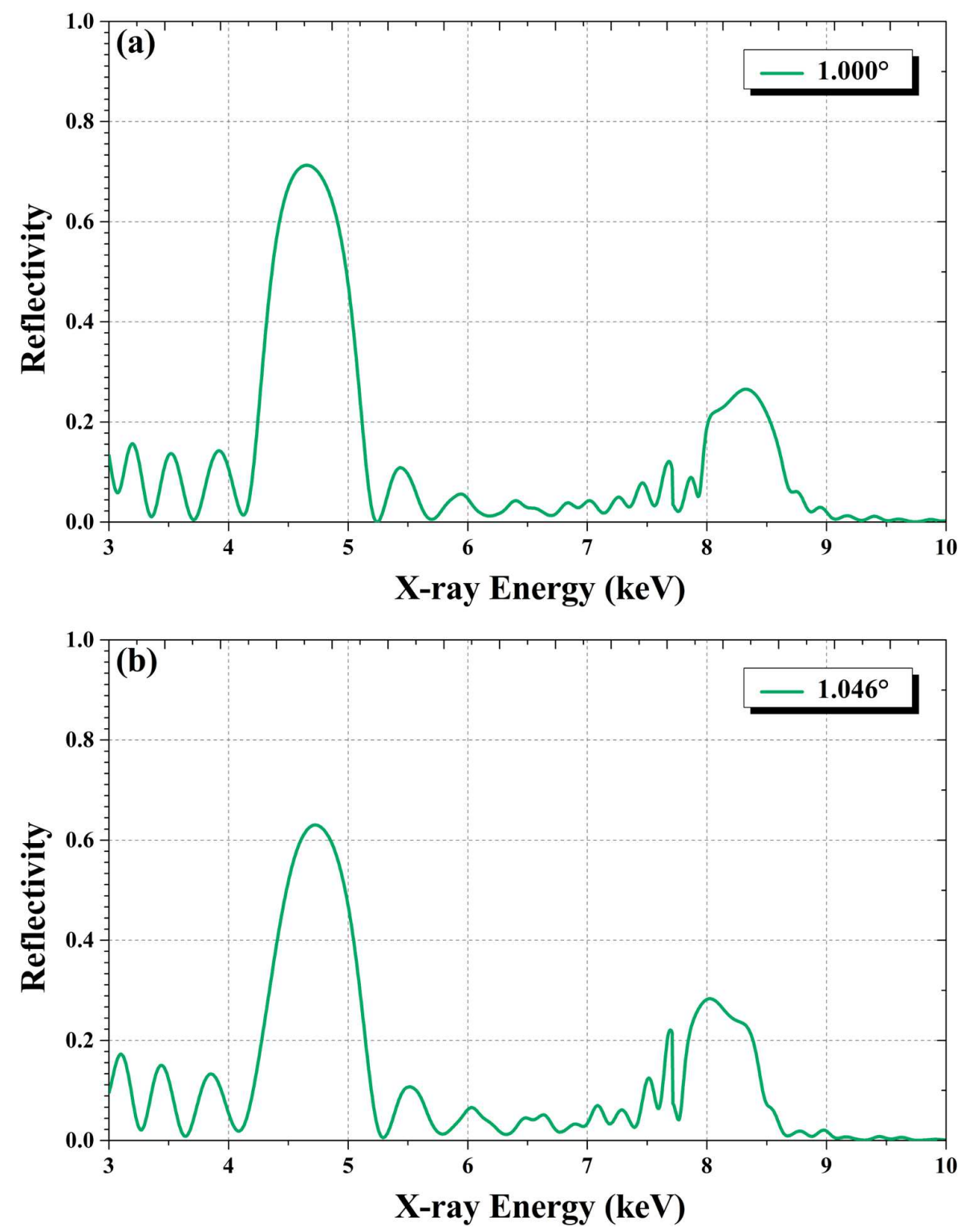

Figure 3. Spectral response curves by fitting the reflectivity measurement results of the KB multilayer for grazing angles of (a) $1.000^{\circ}$ and (b) $1.046^{\circ}$.

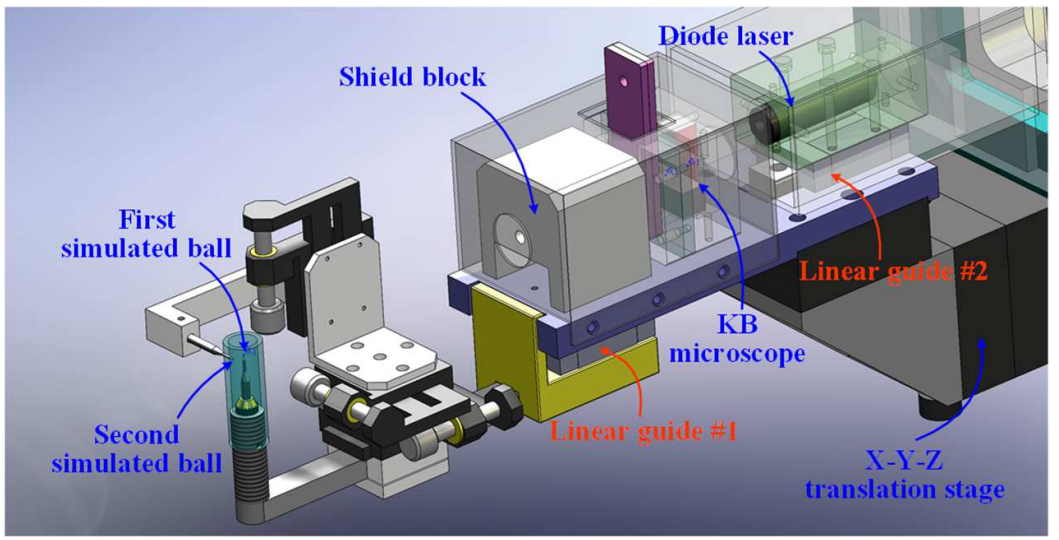

Figure 4. Alignment parts of the multilayer KB microscope based on dual simulated balls. 

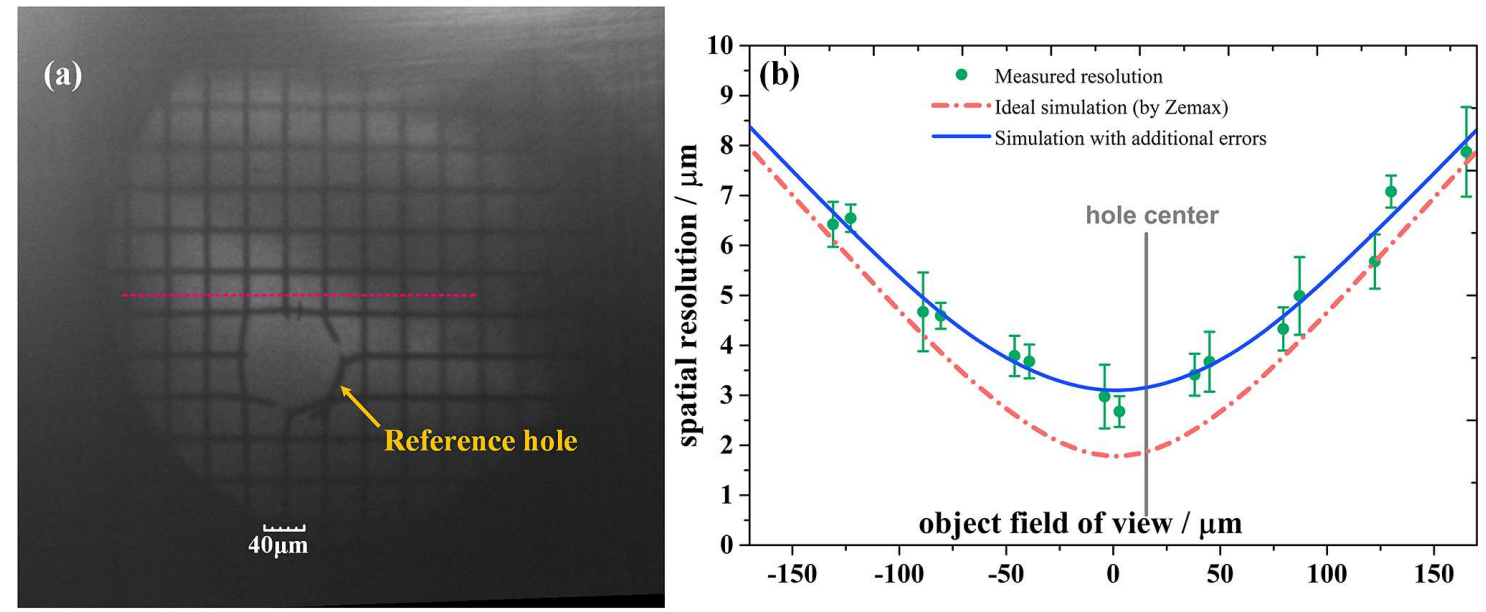

Figure 5. (a) Spatial resolution testing image and (b) calibrated result of the 600-mesh Au grid backlighted by a copper X-ray tube.

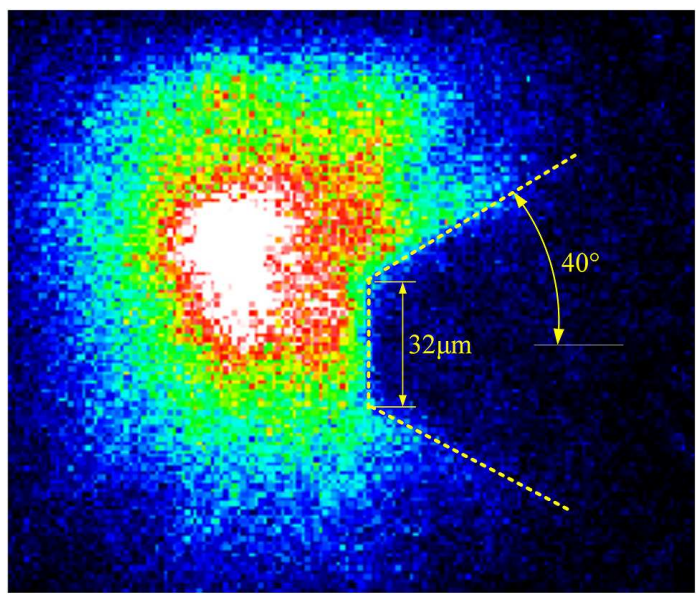

Figure 6. Test result of the KB microscope by Ti flash radiography of the indirect-driven gold cone target.

actual application condition, which was $5.0 \mathrm{~mm}$. In addition, we used a red diode laser (diameter $\sim 1 \mathrm{~mm}$ ) fixed on the stainless-steel plate of the KB microscope to indicate the image point. Both simulated balls were removable from the $\mathrm{KB}$ microscope by two precise linear guides (as shown in Figure 4) with a repeat positioning accuracy of up to $20 \mu \mathrm{m}$. The shielding block at the front of the KB microscope was made of lead-antimony alloy, which is used to shield highenergy background noise.

\section{Experiment on SG-II Update}

The microscope alignment was performed in the following steps at the SG-II Update facility for a fast-ignition study. Under the instruction of the target view system ${ }^{[16]}$, the microscope was aligned by an $x-y-z$ translation stage until the first simulated ball was moved to the desired diagnostic position. The position of the second simulated ball at this time was used as the focus point of the picosecond-pulse laser beam. The image plate (Fuji, BAS-SR2025) was then placed at the position indicated by the red diode laser. Lastly, the ball pointer and the diode laser were removed from two linear guides.

Figure 6 shows the test result of the microscope by Ti flash radiography of the indirect-driven gold cone target. The width of the gold cone top was approximately $32 \mu \mathrm{m}$ with a cone angle of approximately $40^{\circ}$. Affected by the repeated positioning accuracy of linear guide and vacuum pumping after alignment, the brightest position of X-ray backlighter has a deviation of about $20 \mu \mathrm{m}$ from the central area to be diagnosed, and its influence on spatial resolution can be ignored as seen from Figure 5(b). An effective backlighter area of flash radiography with approximately $80 \mu \mathrm{m}$ diameter was produced by the picosecond-pulse laser beam of the SG-II Update facility, which covers the entire area on left side of the cone top that we want to diagnose.

\section{Conclusion}

High-resolution X-ray flash radiography of the Ti characteristic lines with a multilayer $\mathrm{KB}$ microscope has been reported herein. The multilayer design, including $\mathrm{Co} / \mathrm{C}$ and W/C stacks, was optimized to obtain a high reflection efficiency of the Ti characteristic lines of more than $60 \%$ while meeting the precise alignment requirement at the $\mathrm{Cu} \mathrm{K} \alpha$ line. The accurate simultaneous indication of the center field of view and backlighter position was realized by dual simulated balls. A spatial resolution of $3-5 \mu \mathrm{m}$ within $\pm 100 \mu \mathrm{m}$ field of view was achieved in the X-ray experiments. The imaging result of the Au-coned $\mathrm{CH}$ shell target on the SG-II Update demonstrated the feasibility for Ti flash radiography. The microscope may also be suitable for backlit imaging with higher X-ray energies if some improvements are made, such as optimizing the multilayer structure to increase the 
reflectivity for higher X-ray energy or using aspheric mirrors to obtain higher collection efficiency while ensuring the spatial resolution.

\section{Acknowledgments}

This work was supported by the National Natural Science Foundation of China (No. 11805212), National Key Research and Development Program of China (No. 2019YFE03080200), and Fundamental Research Funds for the Central Universities (No. 22120200405).

\section{References}

1. S. Atzeni and J. Meyer-ter-Vehn, The Physics of Inertial Fusion: Beam Plasma Interaction, Hydrodynamics, Hot Dense Matter, International Series of Monographs on Physics (Clarendon Press, Oxford, England, 2004).

2. B. Han, F. Wang, D. Salzmann, J. Zhong, and G. Zhao, High Power Laser Sci. Eng. 9, e9 (2021).

3. L. A. Pickworth, B. A. Hammel, V. A. Smalyuk, A. G. MacPhee, H. A. Scott, H. F. Robey, O. L. Landen, M. A. Barrios, S. P. Regan, M. B. Schneider, M. Hoppe, Jr., T. Konut, D. Holunga, C. Walters, B. Haid, and M. Dayton, Phys. Rev. Lett. 117, 035001 (2016).

4. E. Brambrink, S. Baton, M. Koenig, R. Yurchak, N. Bidaut, B. Albertazzi, J. E. Cross, G. Gregori, A. Rigby, E. Falize, A. Pelka, F. Kroll, S. Pikuz, Y. Sakawa, N. Ozaki, C. Kuranz, M. Manuel, C. Li, P. Tzeferacos, and D. Lamb. High Power Laser Sci. Eng. 4, e30 (2016).

5. A. Morace, L. Fedeli, D. Batani, S. Baton, F. N. Beg, S. Hulin, L. C. Jarrott, A. Margarit, M. Nakai, and M. Nakatsutsumi, Phys. Plasmas 21, 102712 (2014).
6. H. Sawada, S. Lee, T. Shiroto, H. Nagatomo, Y. Arikawa, H. Nishimura, T. Ueda, K. Shigemor, A. Sunahar, and N. Ohnishi, Appl. Phys. Lett. 108, 254101 (2016).

7. C. Stoeckl, T. Filkins, R. Jungquist, C. Mileham, N. R. Pereira, S. P. Regan, M. J. Shoup, and W. Theobald, Rev. Sci. Instrum. 89, 10G124 (2018).

8. L. C. Jarrott, M. S. Wei, C. McGuffey, A. A. Solodov, W. Theobald, B. Qiao, C. Stoeckl, R. Betti, H. Chen, J. Delettrez, T. Döppner, E. M. Giraldez, V. Y. Glebov, H. Habara, T. Iwawaki, M. H. Key, R. W. Luo, F. J. Marshall, H. S. McLean, C. Mileham, P. K. Patel, J. J. Santos, H. Sawada, R. B. Stephens, T. Yabuuchi, and F. N. Beg, Nat. Phys. 12, 499 (2016).

9. W. Theobald, A. A. Solodov, C. Stoeckl, K. S. Anderson, F. N. Beg, R. Epstein, G. Fiksel, E. M. Giraldez, V. Y. Glebov, and H. Habara, Nat. Commun. 5, 5785 (2014).

10. P. Kirkpatrick and A. V. Baez, J. Opt. Soc. Am. 38, 766 (1948).

11. L. A. Pickworth, J. Ayers, P. Bell, N. F. Brejnholt, J. G. Buscho, D. Bradley, T. Decker, S. Hau-Riege, J. Kilkenny, T. McCarville, T. Pardini, J. Vogel, and C. Walton, Rev. Sci. Instrum. 87, 11E316 (2016)

12. F. J. Marshall, R. E. Bahr, V. N. Goncharov, V. Y. Glebov, B. Peng, S. P. Regan, T. C. Sangster, and C. Stoeckl, Rev. Sci. Instrum. 88, 093702 (2017).

13. S. Z. Yi, Z. Zhang, Q. S. Huang, Z. Zhang, Z. S. Wang, L. Wei, D. X. Liu, L. F. Cao, and Y. Q. Gu, Rev. Sci. Instrum. 89, 036105 (2018).

14. W. Theobald, in OMEGA Laser Facility Users' Group Workshop (2009).

15. S. Z. Yi, B. Z. Mu, X. Wang, J. T. Zhu, L. Jiang, Z. S. Wang, and P. F. He, Chin. Opt. Lett. 12, 013401 (2014).

16. L. Ren, P. Shao, D. Zhao, Y. Zhou, Z. Cai, N. Hua, Z. Jiao, L. Xia, Z. Qiao, R. Wu, L. Ji, D. Liu, L. Ju, W. Pan, Q. Li, Q. Ye, M. Sun, J. Zhu, and Z. Lin, High Power Laser Sci. Eng. 6, e10 (2018). 